\title{
Estudo cinético da adsorção de chumbo em biocarvão de Poincianell pluviosa var. peltophoroides (Benth.) L. P. Queiroz
}

Grandes quantidades de poluentes são despejadas em corpos hídricos diariamente por processos agrícolas, químicos e industriais e muito deles, como os metais pesados, não são removidos por tratamentos físico-químicos usuais em estações de tratamento. Existem diversos tipos de tecnologias disponíveis para tratamento de contaminantes perigosos e uma delas é a adsorção, que tem baixo custo e facilidade de operação. Considerando o exposto, o objetivo do presente trabalho fo estudar o potencial de adsorção do chumbo em biocarvão de Poincianella pluviosa var. peltophoroides (Benth.) L. P. Queiroz em amostras aquosas. Resíduos de poda foram preparados termicamente para obtenção do biocarvão e então foram realizados os ensaios cinéticos em um período de $24 \mathrm{~h}$, utilizando $1 \mathrm{~g}$ de adsorvente em contato com $50 \mathrm{~mL}$ de solução de chumbo $100 \mathrm{mg} / \mathrm{L}$. Foram avaliados os modelos de pseudo-primeira ordem e pseudo-segunda ordem aos dados experimentais. Para o estudo cinético foi alcançada uma remoção de $100 \%$, o equilíbrio foi adotado de 360 min e o melhor ajuste cinético foi com o modelo de pseudo-primeira ordem. Com os resultados obtidos pôde-se comprovar a eficácia da adsorção do chumbo por biocarvão feito de resíduo de podas da Poincianella pluviosa var. peltophoroides (Benth.) L. P. Queiroz.

\section{Kinetics study of lead adsorption using Poincianella pluviosa var. Peltophoroides (Benth.) L. P. Queiroz biochar}

\begin{abstract}
Large amounts of polluting substances are dumped daily into water bodies due to agricultural, chemical and industrial processes and many of them, such as heavy metals, are not removed by the usual facilities inf treatment plants. There are several types of technologies available for the treatment of hazardous contaminants and one of them is the adsorption, which presents low cost and easy operation. The objective of this work was to study the potential of lead adsorption by biochar of Poincianella pluviosa var. peltophoroids (Benth.) L. P. Queiroz in aqueous samples. The pruning residues were thermally treated for obtaining biochar and then kinetics tests of 24 hours were performed using $1 \mathrm{~g}$ of the adsorbent previously prepared in contact with a lead solution $100 \mathrm{mg} / \mathrm{L}$. The pseudo-first order and pseudo-second order models were fitted to the experimental data. It was achieved $100 \%$ of lead removal in the kinectics studies, and the equilibrium was adopted in $360 \mathrm{~min}$. The data was better adjusted with pseudo-second-order model. The results obtained were able to prove the adsorption of lead by coal of pruning residues of Poincianella pluviosa var. peltophoroides (Benth.) L. P. Queiroz.
\end{abstract}

Keywords: Lead; Adsorption; Biochar; Poincianella pluviosa var. peltophoroides (Benth.) L. P. Queiroz.

Topic: Desenvolvimento, Sustentabilidade e Meio Ambiente

Reviewed anonymously in the process of blind peer.
Received: 05/10/2020

Approved: $26 / 11 / 2020$
Nelson Consolin Filho (it)

Universidade Tecnológica Federal do Paraná, Brasil

http://lattes.cnpq.br/5820606948533146

http://orcid.org/0000-0002-4847-0701

consolin@utfpr.edu.br

Leonardo Coelho de Oliveira Riboldi (i)

Universidade Tecnológica Federal do Paraná, Brasil

http://lattes.cnpq.br/7673603720707014

http://orcid.org/0000-0002-6377-9969

leo riboldi@hotmail.com

Morgana Suszek Gonçalves (DD

Universidade Tecnológica Federal do Paraná, Brasil

http://lattes.cnpq.br/0497756801594713

http://orcid.org/0000-0002-7576-2937

morgana@utfpr.edu.br
Flávia Vieira da Silva-Medeiros (iD)

Universidade Tecnológica Federal do Paraná, Brasil

http://lattes.cnpq.br/8298141172237555

http://orcid.org/0000-0001-6490-8876

flaviav@utfpr.edu.br
Referencing this:

CONSOLIN FILHO, N.; RIBOLDI, L. C. O.; GONÇALVES, M. S.; MEDEIROS, F. V. S.. Estudo cinético da adsorção de chumbo em biocarvão de Poincianell pluviosa var. peltophoroides (Benth.) L. P. Queiroz. Revista Ibero Americana de Ciências Ambientais, v.11, n.6, p.472-478, 2020. DOI: http://doi.org/10.6008/CBPC2179-6858.2020.006.0038 


\section{INTRODUÇÃO}

A água como fonte de vida é essencial para todos os aspectos da vida humana. No entanto, atualmente países enfrentam escassez de água devido ao rápido crescimento da população, desenvolvimento da indústria, mudanças climáticas, má administração do uso da água, entre outros e, como a escassez e a poluição da água são os principais problemas globais, a gestão é urgentemente necessária, uma vez que contaminantes considerados perigosos entram nos recursos hídricos principalmente via descarga direta de efluentes industriais e atividades urbanas (PUTRO et al., 2017).

A poluição por metais pesados em muitas partes do mundo tem aumentado nas últimas décadas devido a seus crescentes usos nos processos agrícolas, químicos e industriais (MENG et al., 2018). Segundo Alhogbi (2017), despejos industriais podem conter altos níveis de metais pesados, que são altamente tóxicos em contato com o meio ambiente.

Os metais pesados em geral afetam intensamente os seres vivos existentes no ambiente. Segundo Gardiner et al. (2017), foi identificado que a presença destes metais pesados pode influenciar em serviços ecossistêmicos fundamentais, como por exemplo, o controle biológico dentro da chamada agricultura urbana, seus dados apresentam que inimigos naturais expostos a metais pesados sofrem impactos reprodutivos, de desenvolvimento, imunológicos e comportamentais sobre os predadores e parasitoides.

Dentre os metais pesados que causam prejuízos ao meio aquático, é possível destacar o chumbo, considerado altamente tóxico. Um estudo anterior mostrou que, a presença de chumbo no ecossistema afeta drasticamente os órgãos de ostras, principalmente brânquias e glândulas digestivas (MENG et al., 2018). 0 chumbo pode surgir de diversas fontes antrópicas como nas atividades industriais de cerâmica e vidro, tingimento de tecidos, refinarias de petróleo, fabricação de baterias, impressões e mineração (ALHOGBI, 2017).

Atualmente existem muitos tipos de tecnologias disponíveis para tratamento de contaminantes perigosos em ambiente aquático como por exemplo: tratamento biológico aeróbio e anaeróbio, oxidação química avançada, separação por membranas e para efeito deste trabalho, a adsorção. Dentre os materiais adsorventes disponíveis, o biocarvão, é um adsorvente de baixo custo e eficaz no tratamento de águas e águas residuárias, particularmente para a remoção de metais pesados (PUTRO et al., 2017; INYANG et al., 2015). Nesse contexto, é possível enquadrar a poda da Poincianella pluviosa var. peltophoroides (Benth.) $L$. $P$. Queiroz como material de interesse, visto que materiais lignocelulósico tem sido estudado como bioadsorvente e suas aplicações são promissoras.

A Poincianella pluviosa var. peltophoroides (Benth.) L. P. Queiroz, conhecida vulgarmente como Sibipiruna, é uma planta de altura variando entre 8 e $16 \mathrm{~m}$, com tronco de 30 a $40 \mathrm{~cm}$ de diâmetro pertencente à família Fabaceae. Sua origem é muito discutível, ocorrendo na Mata Atlântica do Rio de Janeiro, sul da Bahia e no Pantanal mato-grossense. Sua madeira é moderadamente pesada, dura, de textura média e de média durabilidade natural. Muito empregada na construção civil, estruturas de móveis, para lenha e carvão, sendo atualmente muito empregada na arborização urbana da região Sudeste do país, 
gerando grande quantidade de resíduos sólidos provenientes de suas podas (LORENZI, 2008).

Considerando o exposto, o objetivo do presente trabalho foi estudar o potencial de adsorção do chumbo em biocarvão proveniente de resíduos de poda da Poincianella pluviosa var. peltophoroides (Benth.) L. P. Queiroz em amostras aquosas.

\section{MATERIAIS E MÉTODOS}

As podas de Poincianella pluviosa var. peltophoroides (Benth.) L. P. Queiroz foram coletadas nas dependências da Universidade Tecnológica Federal do Paraná - Campus Campo Mourão. Inicialmente foram selecionados os espécimes saudáveis e então foram podados os galhos com tesoura de poda. Na sequência, as amostras foram trituradas em moinho de facas e secas em estufa de circulação forçada por um período de uma semana a $40 \circ \mathrm{C}$.

$\mathrm{O}$ tratamento térmico foi realizado a partir do uso de um reator pirolítico, em forno mufla a 500 ㄷ durante um período de duas horas. Após o processo de pirólise, o reator foi retirado do forno e mantido em temperatura ambiente por 24 horas para sua posterior abertura. Com o intuito de se aferir a porcentagem de rendimento do biocarvão produzido, foram pesados o material triturado das podas de Poincianella pluviosa var. peltophoroides (Benth.) L. P. Queiroz e o biocarvão obtido, antes e após o processo de pirólise, respectivamente.

Para a utilização do biocarvão como adsorvente no experimento de estudo cinético, anteriormente o mesmo passou por sucessivas lavagens com água destilada e secagem a 40 ํㅡ em estufa de circulação forçada.NA solução de trabalho foi preparada a partir de Nitrato de Chumbo P.A. em água destilada na concentração de $100 \mathrm{mg} / \mathrm{L}$ de chumbo. $\mathrm{O}$ pH da solução foi ajustado para 3, buscando-se prevenir a precipitação do chumbo. Para o ajuste de pH foram utilizadas soluções de $1 \mathrm{~mol} / \mathrm{L}$ de $\mathrm{HNO}_{3}$ ou $1 \mathrm{~mol} / \mathrm{L}$ de $\mathrm{NaOH}$.

Os estudos cinéticos foram realizados em duplicata, adaptando-se a metodologia utilizada por Zhou et al. (2017). Sendo assim, 11 amostras de $1 \mathrm{~g}$ do biocarvão foram colocadas em contato com $50 \mathrm{~mL}$ da solução de chumbo em erlenmeyers de $100 \mathrm{~mL}$, os quais permaneceram em agitação a $150 \mathrm{rpm}$ e a $35^{\circ} \mathrm{C}$ por um período total de tempo de contato de 24 horas. Em intervalos de tempo pré-definidos foram retiradas amostras para realização da determinação de chumbo na solução por espectrofotometria de absorção atômica (Analytik Jena - novAA ${ }^{\circledR} 300$ ).

A eficiência de remoção do chumbo $(R, \%)$ e a capacidade de adsorção do biossorvente $\left(q_{e}\right)$ foram determinadas a partir das Equações (1) e (2):

$$
\begin{array}{ll}
R=\frac{C_{0}-C_{e}}{C_{0}} \times 100 \% & \text { Equação (1) } \\
q_{e}=\frac{\left(C_{0}-C_{e}\right) V}{m} & \text { Equação (2) }
\end{array}
$$


$\mathrm{V}=$ Volume da solução (L); $\mathrm{C}_{0}=$ Concentração inicial de chumbo $\left(\mathrm{mg} . \mathrm{L}^{-1}\right) ;$ $\mathrm{C}_{\mathrm{e}}=$ Concentração de chumbo depois da adsorção $\left(\mathrm{mg} \cdot \mathrm{L}^{-1}\right)$; $\mathrm{m}=$ Massa de adsorvente $(\mathrm{g})$.

Os dados obtidos no teste cinético foram então ajustados aos modelos de pseudo-primeira ordem, proposto inicialmente por Lagergren (1898) e de pseudo-segunda ordem como proposto por Ho et al. (1999), de acordo com as equações (3) e (4).

$$
\begin{aligned}
& q_{t}=q_{e}\left[1-e^{-k_{1} t}\right] \\
& q_{t}=\frac{k_{2} q_{e}^{2} t}{1+k_{2} q_{e} t}
\end{aligned}
$$$$
\begin{array}{r}
\text { Equação (3) } \\
\begin{array}{r}
\text { Em que: } \\
\mathrm{q}_{\mathrm{t}}=\text { Capacidade de adsorção no tempo } \mathrm{t}\left(\mathrm{mg} \cdot \mathrm{g}^{-1}\right) ; \\
\mathrm{q}_{\mathrm{e}}=\text { Capacidade de adsorção no equilíbrio }\left(\mathrm{mg} \cdot \mathrm{g}^{-1}\right) ; \\
\mathrm{t}=\text { Tempo de reação }(\mathrm{min}) ;
\end{array} \\
\mathrm{k}_{1}=\text { Constante de velocidade de adsorção de pseudo-primeira ordem }\left(\mathrm{min}^{-1}\right) .
\end{array}
$$$$
\text { Equação (4) }
$$

Em que:

qt: Capacidade de adsorção no tempo t $\left(\mathrm{mg}^{\mathrm{g}} \mathrm{g}^{-1}\right)$; $q_{\mathrm{e}}$ : Capacidade de adsorção no equilíbrio $\left(\mathrm{mg} \cdot \mathrm{g}^{-1}\right)$; t: Tempo de reação $(\mathrm{min})$; k2: Constante de velocidade de adsorção de pseudo-segunda ordem (g. $\left.\mathrm{mg}^{-1} \cdot \mathrm{min}^{-1}\right)$.

O modelo que melhor representou os dados experimentais foi escolhido a partir da análise do coeficiente de determinação $\left(R^{2}\right)$ e pela comparação entre o qe calculado e o qe experimental dos modelos.

\section{RESULTADOS E DISCUSSÃO}

O processo de pirólise para obtenção do biocarvão (adsorvente) utilizado neste trabalho obteve rendimento de 41,5\%. De acordo com Demirbas (2004), a relação entre temperatura e rendimento tem particularidades em cada material e pode ser influenciada segundo a quantidade de celulose, hemicelulose e lignina. Conz (2016) encontrou rendimento de 42,41\% para o biocarvão produzido a partir de serragem para temperatura de pirólise de $450^{\circ} \mathrm{C}$, valor aproximado ao deste trabalho.

A curva cinética (Figura 1) demonstrou remoção máxima de 100\% aos 720 min, que se mantém até o final do experimento. É possível observar que entre 120 e 180 min a adsorção apresenta baixa variação, apenas 1\% aproximadamente, porém entre 180 min e 360 min ocorreu uma alta variação, de 43\% até 95\% de remoção. A partir deste tempo a variação, mesmo alcançando $100 \%$ de remoção, não foi significativa sendo assim adotado o tempo ótimo de remoção de 6 horas (360 min).

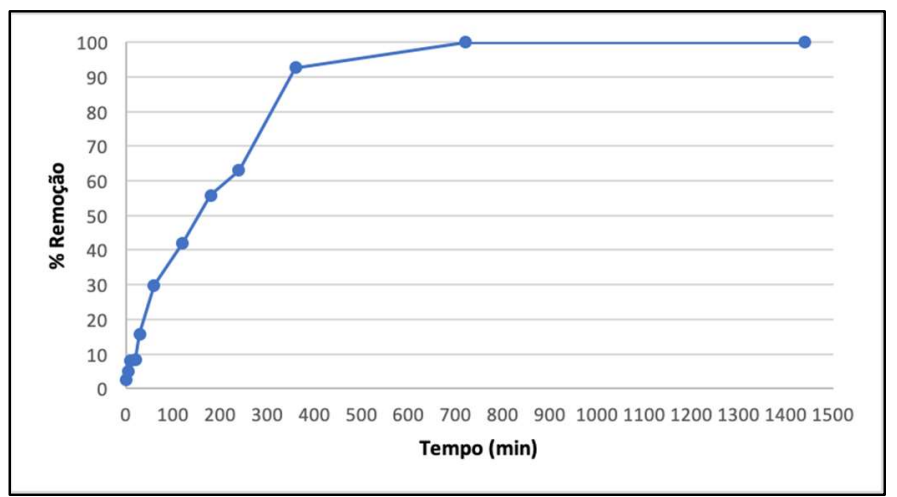

Figura 1: Curva cinética da remoção de Pb em biocarvão de Poincianella pluviosa var. peltophoroides (Benth.) L. $P$. Queiroz 
Como observado por Majumdare et al. (2010) e Panda et al. (2006) em trabalhos de adsorção de metais em biomassa de Mucor rouxii e Lathyrus sativus, respectivamente, a adsorção foi mais rápida no início do estudo cinético, ocorrendo majoritariamente nos primeiros 10 minutos e atingindo o equilíbrio em 360 minutos. De acordo com estes autores, a taxa inicial mais rápida pode ser devida a abundância de sítios disponíveis para a ligação do chumbo na superfície dos biossorventes utilizados, seguida então de uma taxa mais baixa até que o equilíbrio fosse atingido.

Em trabalho similar realizado por Alhogbi (2017), foi avaliada a adsorção de chumbo utilizando a casca do café utilizando 0,3 a 2 g de adsorvente em uma solução com concentração inicial de 400 mg/L, e obteve um tempo ótimo de adsorção de 60 minutos com remoção de $98 \%$.

Zhou et al. (2018) estudou a adsorção de chumbo utilizando resíduos têxteis enxertados com ácido acrílico a uma concentração de $10 \mathrm{mg} / \mathrm{L}, 1 \mathrm{~g}$ de adsorvente, em pH 7 e temperatura de 25 으, e alcançou o equilíbrio em 30 min de adsorção, chegando a uma porcentagem de remoção de 95,3\%. Em comparação aos dados encontrados no presente estudo, diferenciando apenas o $\mathrm{pH}$ e a temperatura, é observado que a eficiência encontrada é similar porém em um tempo efetivamente menor, porém neste não foi atingido $100 \%$ de remoção.

Os dados experimentais foram ajustados às formas lineares dos modelos cinéticos dados pelas equações de pseudo-primeira ordem (Eq. 3) e de pseudo-segunda ordem (Eq. 4), baseados na capacidade de equilíbrio. Os resultados encontrados estão dispostos na Tabela 1.

Tabela 1: Parâmetros dos modelos cinéticos de pseudo-primeira ordem e pseudo-segunda ordem aos dados experimentais

\begin{tabular}{|c|c|c|c|c|c|c|}
\hline & Pseudo $p$ & ordem & & Pseudo s & ordem & \\
\hline $\begin{array}{l}q_{e}(\text { exp. }) \\
\left(\mathrm{mg}^{-1} \mathrm{~g}^{-1}\right)\end{array}$ & $\begin{array}{l}\mathrm{q}_{\mathrm{e}} \text { (calc.) } \\
\text { (mg/g) }\end{array}$ & $\begin{array}{l}\mathrm{k}_{1} \\
\left(\min ^{-1}\right)\end{array}$ & $\mathrm{R}^{2}$ & $\begin{array}{l}\mathrm{q}_{\mathrm{e}} \text { (calc.) } \\
\left(\mathrm{mg}^{-1} \mathrm{~g}^{-1}\right)\end{array}$ & $\begin{array}{l}\mathrm{k}_{2} \\
\left(\mathrm{~g} \cdot \mathrm{mg}^{-1} \cdot \mathrm{min}^{-1}\right)\end{array}$ & $\mathrm{R}^{2}$ \\
\hline 0,475 & 0,465 & 0,00414 & F0,9601 & 0,565 & 0,011 & 00,9715 \\
\hline
\end{tabular}

Os coeficientes de correlação foram altos para os dois modelos avaliados, porém foi maior para o modelo de pseudo-segunda ordem, o que poderia indicar que o processo de adsorção proposto segue este mecanismo. Contudo, o coeficiente de correlação, mesmo que importante, não é decisivo a qual modelo os dados se ajustam (LIU et al., 2010; ZAIDI et al., 2018). Nota-se que o valor de $\mathrm{q}_{\mathrm{e}}$ experimental $(0,475 \mathrm{mg} / \mathrm{g}$ ) se aproxima mais ao $\mathrm{q}_{\mathrm{e}}$ calculado pelo modelo de pseudo-primeira ordem $(0,465 \mathrm{mg} / \mathrm{g})$ do que ao calculado pelo modelo de pseudo-segunda ordem $(0,565 \mathrm{mg} / \mathrm{g})$, o que sugere que o modelo proposto por Lagergren (1898) descreve melhor a adsorção do chumbo em biocarvão de Poincianella pluviosa var. peltophoroides (Benth.) L. P. Queiroz.

O modelo de pseudo-primeira ordem tem como pressuposto que a etapa limitante da velocidade da adsorção é a fisiossorção (LAGERGREN, 1898). Segundo Nascimento et al. (2014), os processos de fisiossorção e quimiosorção se diferem pois, no caso da fisiossorção a ligação do adsorbato com a superfície do adsorvente envolve uma interação relativamente fraca normalmente atribuída às forças de Van der Waalls, que são similares às forças de coesão molecular, já a quimiossorção, resulta em uma ligação química por ocorrer a troca ou partilha de elétrons entre as moléculas do adsorvato e a superfície do adsorvente, portanto 
bem mais forte que a fisiossorção.

Sendo assim, o alto coeficiente de correlação observado $(0,9601)$ e o valor do $q_{e}$ experimental calculado $(0,465 \mathrm{mg} / \mathrm{g})$ sugerem que o processo de adsorção de chumbo em biocarvão de Poincianella pluviosa var. peltophoroides (Benth.) L. P. Queiroz é dominado pelo mecanismo de pseudo-primeira ordem, logo, controlado pelo processo de fisiossorção (LIU et al., 2010; ZAIDI et al., 2018).

\section{CONCLUSÕES}

Tendo em conta os resultados obtidos no presente estudo, pode-se comprovar alto potencial de adsorção do chumbo pelo biocarvão proveniente de poda proveniente da Poincianella pluviosa var. peltophoroides (Benth.) L. P. Queiroz, alcançando uma taxa de remoção de 100\%.

Sobre o estudo cinético, foi possível observar que o tempo ótimo de remoção de chumbo foi de 6 horas e o modelo que se ajustou melhor os dados experimentais foi o de pseudo-primeira ordem.

Os resíduos de poda da Poincianella pluviosa var. peltophoroides (Benth.) L. P. Queiroz são enquadrados como resíduos sólidos urbanos e esta espécie arbórea é comumente encontrada na arborização urbana em todo o Brasil, assim além da alta eficiência na remoção do chumbo, este adsorvente é facilmente encontrado, agregando assim maior valor pelo seu aproveitamento e utilização na fabricação de biocarvões usados como adsorvente.

\section{REFERÊNCIAS}

ALHOGBI, B. G.. Potential of coffee husk biomass waste for the adsorption of $\mathrm{Pb}$ (II) ion from aqueous solutions. Sustainable Chemistry and Pharmacy, v.6, n.21, 2017.

CONZ, R. F.. Dissertação de Mestrado. São Paulo: Universidade de São Paulo, 2015.

DEMIRBAS, A.. Effects of temperature and particle size on bio-char yield from pyrolysis of agricultural residues. Journal of Analytical and Apllied Pyrolysis, v.72, n.243, 2004.

GARDINER, M. M.; HARWOOD, J. D.. Influence of heavy metal contamination on urban natural enemies and biological control. Current Opinion In Insect Science, v.20, n.45, 2017.

HO, Y. S.; MCKAY, G.. Pseudo-second order model for sorption processes. Process Biochemistry, v.34, p.451, 1999

INYANG, M. I.; GAO, B.; YAO, Y.; XUE, Y.; ZIMMERMAN, A.; MOSA, A.; PULLAMMANAPPALLIL, P.; OK, Y. S.; CAO, X.. A review of biochar as a low-cost adsorbent for aqueous heavy metal removal. Critical Reviews In Environmental Science and Technology, v.46, n.406, 2015.

LAGERGREN, S.. About the theory of so-called adsorption of soluble substances. Kungliga Svenska

Vetenskapsakademiens, Handlingar, v.24, n.1, 1898

LIU, Y.; WANG, W.; WANG, A.. Adsorption of lead ions from aqueous solution by using carboxymethyl cellulose-g-poly (acrylic acid)/attapulgite hydrogel composites. Desalination, v.259, n.258, 2010.

LORENZI, H.. Árvores brasileiras: Manual de identificação e cultivo de plantas arbóreas nativas do Brasil. 5 ed. Plantarum: Nova Odessa, 2008.

MAJUMDARE, S. S.; DAS, S. K.; CHAKRAVARTY, R.; SAHA, T.; BANDYOPADHYAY, T. S.; GUHA, A. K.. A study on lead adsorption by Mucor rouxii biomass. Desalination, 251, 96, 2010.

MENG, J.; WANG, W.-X.; LI, L.; ZHANG, G.. Tissue-specific molecular and cellular toxicity of $\mathrm{Pb}$ in the oyster (Crassostrea gigas): mRNA expression and physiological studies. Aquatic Toxicology, v.198, n.257, 2018.

MENG, L.; GAO, J.; ZHONG, Y.; WHANG, Z.; CHEN, K.; GUO, Z.. Supergravity separation for recovering $\mathrm{Pb}$ and $\mathrm{Sn}$ from electronic waste. Separation and Purification Technology v.191, n.375, 2018.

NASCIMENTO, R. F.; LIMA, A. C. A.; VIDAL, C. B.; MELO, D. Q.; RAULINO, G. S. C.. Adsorção: aspectos teóricos e aplicações ambientais. Fortaleza: Imprensa Universitária, 2014.

PANDA, G. C.; DAS, S. K; CHATTERJEE, S.; MAITY, P. B.; BANDOPADHYAY, T. S.; GUHA, A. K.. Adsorption of cadmium on husk of Lathyrus sativus: Physico-chemical study. Colloids Surface, B Biointerfaces, v.50, n.49, 2006.

PUTRO, J. N.; KURNIAWAN, A.; ISMADJI, S.; JU, Y.-H. Nanocellulose based biosorbents for wastewater treatment: 
Study of isotherm, kinetic, thermodynamic and reusability. Environmental Nanotechnology, Monitoring \& Management, v.8, n.134, 2017.

ZAIDI, N. A. H. M.; LIM, L. B. L.; USMAN, A.. Enhancing adsorption of $\mathrm{Pb}$ (II) from aqueous solution by $\mathrm{NaOH}$ and EDTA modified Artocarpus odoratissimus leaves. Journal Of
Environmental Chemical Engineering, v.6, n.7172, 2018

ZHOU, T.; XIA, F.; DENG, Y.; ZHAO, Y.. Removal of Pb(II) from aqueous solutions using waste textiles/poly(acrylic acid) composite synthesized by radical polymerization technique. Journal of Environmental Sciences, v.67, n.368, 2018.

A CBPC - Companhia Brasileira de Produção Científica (CNPJ: 11.221.422/0001-03) detém os direitos materiais desta publicação. Os direitos referem-se à publicação do trabalho em qualquer parte do mundo, incluindo os direitos às renovações, expansões e disseminações da contribuição, bem como outros direitos subsidiários. Todos os trabalhos publicados eletronicamente poderão posteriormente ser publicados em coletâneas impressas sob coordenação da Sustenere Publishing, da Companhia Brasileira de Produção Científica e seus parceiros autorizados. Os (as) autores (as) preservam os direitos autorais, mas não têm permissão para a publicação da contribuição em outro meio, impresso ou digital, em português ou em tradução. 